\title{
Efficacy and safety of continuous infusions with elastomeric pumps for outpatient parenteral antimicrobial therapy (OPAT): an observational study
}

\author{
Rachel Voumard ${ }^{1}$, Céline Gardiol ${ }^{2}$, Pascal André ${ }^{3}$, Lyne Arensdorff ${ }^{1}$, Camille Cochet ${ }^{1}$, Noémie Boillat-Blanco ${ }^{2}$, \\ Laurent Decosterd ${ }^{4}$, Thierry Buclin ${ }^{3}$ and Serge de Vallière ${ }^{2 *}$ \\ ${ }^{1}$ Department of Ambulatory Care and Community Medicine, University Hospital of Lausanne, Av du Bugnon 44, 1011 Lausanne, \\ Switzerland; ${ }^{2}$ Department of Ambulatory Care and Community Medicine, and Infectious Diseases Service, University Hospital of \\ Lausanne, Av du Bugnon 44, 1011 Lausanne, Switzerland; ${ }^{3}$ Service of Clinical Pharmacology, University Hospital of Lausanne, \\ Av du Bugnon 17, 1011 Lausanne, Switzerland; ${ }^{4}$ Laboratory and Service of Clinical Pharmacology, University Hospital of Lausanne, \\ Av du Bugnon 19, 1011 Lausanne, Switzerland
}

${ }^{*}$ Corresponding author. Tel: +41-79-556-43-12; Fax: +41-21-314-41-79; E-mail: serge.de-valliere@hospvd.ch

Received 13 December 2017; returned 9 February 2018; revised 7 May 2018; accepted 19 May 2018

\begin{abstract}
Objectives: This study aimed to evaluate the efficacy and safety of continuous antimicrobial infusion using elastomeric pumps in an outpatient setting, while simultaneously documenting circulating antibiotic concentration exposure achieved with this mode of administration.

Methods: Clinical outcomes, adverse events and antibiotic plasma concentrations were recorded for all patients treated by continuous infusion with elastomeric pumps at the outpatient parenteral antimicrobial therapy (OPAT) unit of the University Hospital of Lausanne between December 2013 and January 2017. The study was registered under ClinicalTrials.gov identifier NCT03221140.

Results: One hundred and fifty outpatients were treated by continuous intravenous infusions using flucloxacillin (70 patients), cefepime (36), vancomycin (32) and piperacillin/tazobactam (12). The calculated free fractions of each antibiotic were above the epidemiological cut-off values for resistance (ECOFF) of the treated microorganisms in $92 \%$ of measurements. Cure was achieved in 143 patients (95\%) 3 months after the end of treatment. Four patients needed unexpected readmission and three had a relapse. In none of the patients with unsuccessful treatment was the ratio of free antibiotic plasma concentration/ECOFF $<1$. Sixteen patients $(11 \%)$ had an adverse event, none of them being of severity grade 4 or 5.
\end{abstract}

Conclusions: Continuous infusions of flucloxacillin, cefepime, vancomycin and piperacillin/tazobactam using elastomeric pumps seem to be an effective and safe approach to treat outpatients. The number of treatment successes was very high and adverse events occurred at a similar rate as reported by other OPAT centres. The measured antibiotic plasma concentrations confirmed adequate drug concentration exposure for the vast majority of patients.

\section{Introduction}

Some patients with difficult-to-treat infections require intravenous antibiotics, often for a prolonged duration, but are otherwise well enough to be treated as outpatients. Considering the numerous advantages of ambulatory treatment, outpatient parenteral antimicrobial therapy (OPAT) centres were initially established in the USA and the concept has now spread to many other countries, notably in Europe.

In this context, elastomeric pumps allow for the continuous infusion of antibiotics with time-dependent killing mechanisms and short half-lives, which would otherwise require several injections per day. As the pumps are changed just once a day, either by the patient himself or by a nurse, it allows a greater autonomy for the patient and decreases the burden on the healthcare system. It avoids multiple daily interventions by the nurses of the OPAT unit or home healthcare services. In some instances, it enables treatment continuation with a first-line agent, which is otherwise difficult to administer on an outpatient basis without a pump. It is probably cost-effective, although a formal economic evaluation still has to be done. Finally, in a previous study, we showed that acceptance and satisfaction was very high among patients receiving antibiotics via elastomeric pumps. ${ }^{1}$ 
The potential degradation of the antibiotics in these devices limits their use. The manufacturers of elastomeric pumps have published antibiotic stability data and most reference documents and guidelines are based on these data from the manufacturers. ${ }^{2}$ However, there are several limitations to these stability data. First, there has been almost no independent verification of these data. Secondly, the data were generated under standardized laboratory conditions, which do not necessarily reflect real-life situations. Thirdly, the tests did not always evaluate antimicrobial stabilities at concentrations and at time points relevant to clinical situations. The BSAC therefore concluded that stability data for all major, most frequently used antibiotics administered via elastomeric pumps are insufficient. ${ }^{3}$

In a previous study, we evaluated the temperature variations of solutions in elastomeric pumps under real-life conditions and showed that these temperatures can exceed $30^{\circ} \mathrm{C} .^{4}$ In the same study we also measured the degradation of flucloxacillin, cefazolin, cefepime and piperacillin/tazobactam in elastomeric pumps worn under real-life conditions. We concluded that the degradation of these antibiotics was acceptable despite the occurrence of excessive temperatures.

The aim of this study was to evaluate the efficacy and safety of continuous infusions with elastomeric pumps for OPAT and to measure circulating antibiotic concentration exposure achieved with this mode of administration.

\section{Methods}

We prospectively collected data from all patients treated by continuous infusion with elastomeric pumps by the OPAT unit of the University Hospital of Lausanne between December 2013 and January 2017. We obtained informed consent for all patients. An analysis of the patients treated by continuous infusions of amoxicillin using elastomeric pumps was published previously and these patients were therefore not included in this report. ${ }^{5}$

Elastomeric pumps of the brand Easypump II 270-27 (B. Braun, Melsungen, Germany) were prepared under laminar flow by the staff of a single pharmacy. Pumps were prepared for up to 7 days and patients were instructed to keep them in their fridge before use. A PICC-line (Power Picc; Becton Dickinson, Eysins, Switzerland) was used for venous access in all patients. An infectious disease specialist evaluated the patients weekly or more frequently if indicated. Patients were encouraged to change their elastomeric pumps by themselves (self-administration). OPAT nurses or home healthcare nurses changed the pumps only if the patient was reluctant or if the health professional considered the patient unable to do selfadministration.

Socio-demographic and clinical data were recorded, namely gender, age, site of infection (osteo-articular; endovascular; urinary; pulmonary; catheter related; abdominal; skin and soft tissue; ear, nose and throat; (NS), microorganisms responsible of the infection, antimicrobial treatment (flucloxacillin, cefepime, vancomycin, piperacillin/tazobactam, other), type of administration (self-administration, administration by a home healthcare nurse, administration at the OPAT clinic, mixed) and duration of treatment.

Continuous infusion was started $1 \mathrm{~h}$ after a loading dose or $1 \mathrm{~h}$ after the last intermittent dose administered at the hospital. We measured antibiotic plasma concentrations after at least $48 \mathrm{~h}$ of continuous infusion. As continuous infusion is expected to generate a steady concentration plateau, we measured plasma concentration at unselected times during treatment. Blood was drawn at the OPAT unit once a week or more frequently in the case of discrepant values or unstable renal function. Antimicrobial drug concentrations in plasma were measured by a validated method of LC coupled to tandem MS using stable isotopically labelled internal standards and matrix-matched calibration samples. For each patient with an identified infectious agent, we calculated the ratio of antibiotic plasma concentration corrected for the free fraction of the antibiotic, divided by the epidemiological cut-off value of resistance (ECOFF) of the bacteria treated. The plasma-free fractions of antibiotics used in these calculations were extracted from the summaries of product characteristics and were as follows: flucloxacillin $=10 \%$, vancomycin $=70 \%$, cefepime $=80 \%$ and piperacillin $=80 \%$. The ECOFF values were extracted from the EUCAST website. $^{6}$

We assessed outcomes at the end of OPAT treatment and 3 months later using the hospital records. The patients were considered cured in the case of absence of fever, no local signs of infection at the end of the treatment as assessed by an infectious disease specialist and no unplanned readmission to our hospital for the same cause within 3 months after the end of treatment. Unplanned readmissions during OPAT, relapses of infection during or after the end of OPAT, or deaths during or within the 3 months after the end of OPAT were considered to be treatment failures. Expected readmissions, such as, e.g. for an elective change of a prosthesis, were not considered treatment failures.

Adverse events were classified according to the Safety Reporting Requirements for INDs and BA/BE Studies FDA Guidance. ${ }^{7}$ Grade classification (grades 1-5) was used as recommended by the Common Terminology Criteria for Adverse Events. ${ }^{8}$ We recorded adverse events during treatment and for the following 3 months.

All analyses were descriptive. The data were collected in Microsoft Excel and analysed using Stata 14.0, through univariate analyses. Graphs were designed using GraphPad 6.0. Ethical approval was granted by the Ethics Committee of the Canton of Vaud (protocol number 34/14). The study was registered under ClinicalTrials.gov identifier NCT03221140.

\section{Results}

Among the 545 patients treated at the OPAT unit during the study period, 150 were included in the analysis (Figure 1). We excluded 395 patients for the following reasons: 366 were treated with antibiotics other than flucloxacillin, cefepime, vancomycin or piperacillin/tazobactam; nine were still on treatment at the time of the study period; and 20 did not receive the antibiotics by continuous infusion.

The 150 included patients treated by continuous intravenous infusions were mostly men (72\%), with a median age of 59 years (range $=16-93$ ). Table S1 (available as Supplementary data at JAC Online) shows the microorganisms involved in the infections. Table 1 summarizes the sites of infection and details of the treatment. Of note, 79 patients (53\%) were treated for osteo-articular infections. The patients were treated with flucloxacillin (70 patients), cefepime (36), vancomycin (32) and piperacillin/tazobactam (12). Duration of treatment varied from 2 to 104 days, with a median of 13 days. Self-administration was performed by $82 \%$ of the patients. Treatment was administered by home healthcare nurses (13\%) or by nurses at the OPAT unit $(4 \%)$ for patients unable or unwilling to do self-administration.

One hundred and forty-three patients (95\%) were cured 3 months after the end of treatment. There were four unexpected readmissions during treatment and three relapses within 3 months after treatment completion. Table 2 shows the characteristics and the type of infections of these patients considered to have experienced treatment failure.

Two hundred and twelve plasma antibiotic concentrations were measured in 101 patients and the mean concentrations 

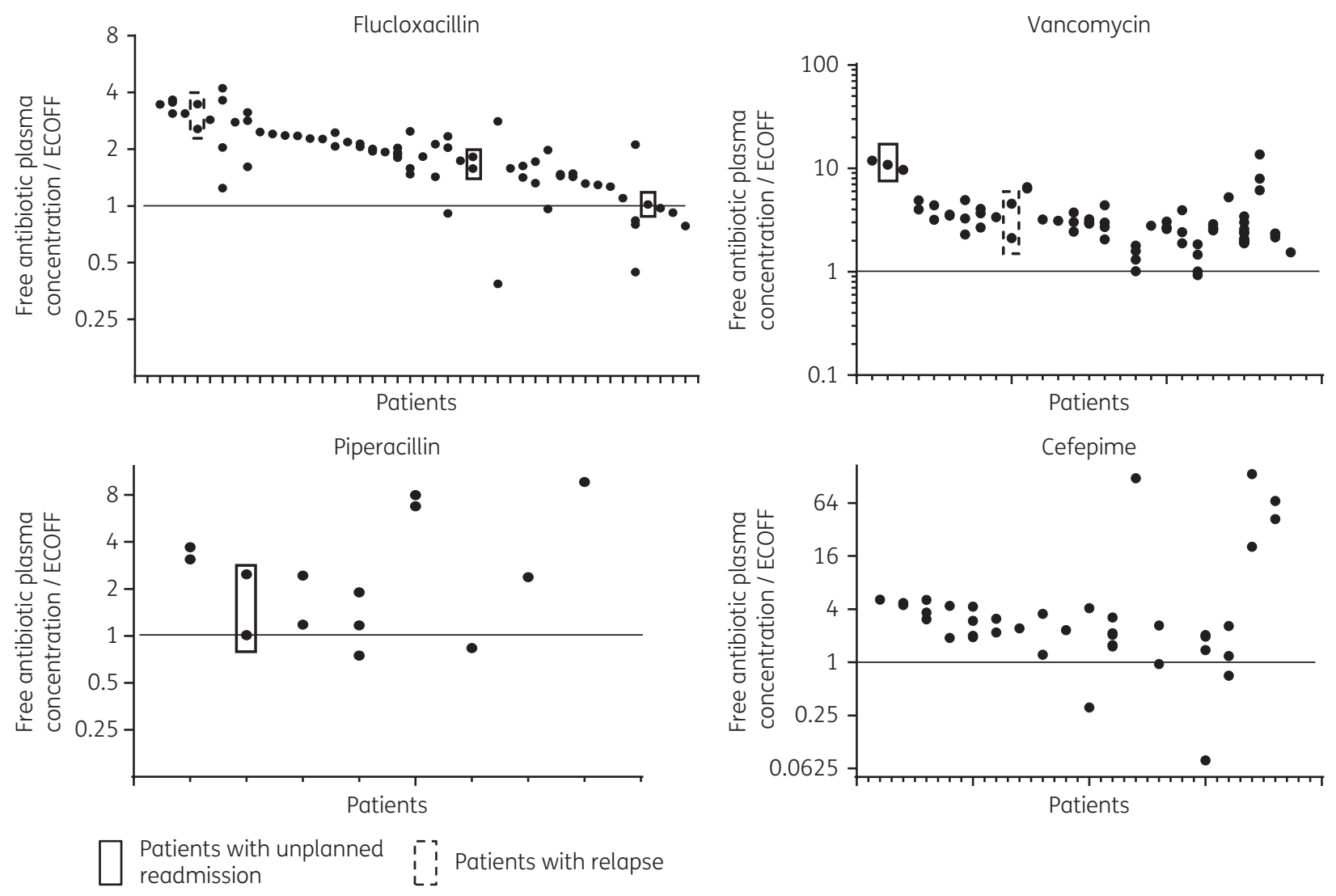

Figure 1. Ratio of the free antibiotic plasma concentration over the ECOFF of the bacteria to be treated.

Table 1. Characteristics of the patients and their treatment

\begin{tabular}{lc}
\hline Male, \% ( $n$ ) & $72(108)$ \\
Age (years), median (range) & $59(16-93)$ \\
Site of infection, \% ( $n$ ) & \\
osteo-articular & $53(79)$ \\
endovascular & $12(18)$ \\
urinary & $11(16)$ \\
pulmonary & $9(13)$ \\
catheter related & $5(8)$ \\
abdominal & $3(5)$ \\
skin and soft tissue & $3(5)$ \\
ear, nose and throat & $3(4)$ \\
CNS & $1(2)$ \\
Antibiotics used, \% ( $n$ ) & \\
flucloxacillin & $47(70)$ \\
cefepime & $24(36)$ \\
vancomycin & $21(32)$ \\
piperacillin/tazobactam & $8(12)$ \\
Administration of antibiotics, \% (n) & \\
self-administration & $82(123)$ \\
home health nurse & $13(19)$ \\
OPAT unit & $4(6)$ \\
mixed & $1(2)$ \\
Duration of treatment (days), median (range) & $13(2-104)$
\end{tabular}

$( \pm$ SD) for each antibiotic were as follows: flucloxacillin $=36 \mathrm{mg} / \mathrm{L}$ ( \pm 15.2$)$, cefepime $=21.3 \mathrm{mg} / \mathrm{L}( \pm 12.1)$, vancomycin $=17.2 \mathrm{mg} / \mathrm{L}$ $( \pm 5.3)$ and piperacillin $=25.8 \mathrm{mg} / \mathrm{L}( \pm 15.7)$. Figure 1 shows the ratio of the calculated free antibiotic plasma concentrations divided by the ECOFF of the microorganisms treated. This ratio was $\geq 1$ for 180 of 196 measurements (92\%): flucloxacillin 62 of $71(87 \%)$, vancomycin 70 of 71 (99\%), cefepime 36 of 40 (90\%) and piperacillin 12 of 14 (86\%). Ten plasma drug concentrations were measured in six of the seven patients who experienced a treatment failure. The ratio of free antibiotic plasma concentrations/ECOFF was $\geq 1$ for all the measurements in these six patients.

Among the 150 patients enrolled, 16 patients (11\%) experienced an adverse event (Table S2), which included 2 cases of grade 3 , namely hospitalization for hypokalaemia and febrile agranulocytosis. The other adverse events were 3 cases of grade 2 ( 2 cases of catheter-related thrombosis and 1 catheter-related infection) and 11 cases of grade 1 [neutropenia (four cases), rash (two), cholestasis (one), thrombocytosis (one), catheter-related superficial thrombosis (one), diarrhoea (one) and renal failure (one)]. None of the adverse events were of grade 4 or 5.

\section{Discussion}

Elastomeric devices have mainly been used for the ambulatory administration of oncological treatments. Several guidelines mention their possible use in the context of OPAT. ${ }^{9,10}$ The use of elastomeric pumps facilitates the ambulatory management of patients and 
Table 2. Patients with treatment failure (unplanned readmission during treatment or relapse of infection within 3 months of the end of treatment)

\begin{tabular}{|c|c|c|c|c|c|c|c|}
\hline Sex & $\begin{array}{c}\text { Age } \\
\text { (years) }\end{array}$ & $\begin{array}{l}\text { Type of } \\
\text { infection }\end{array}$ & Microorganism & $\begin{array}{l}\text { Days of } \\
\text { OPAT }\end{array}$ & Antibiotic & $\begin{array}{l}\text { Type of OPAT } \\
\text { failure }\end{array}$ & $\begin{array}{l}\text { Management } \\
\text { of failure }\end{array}$ \\
\hline Female & 59 & $\begin{array}{l}\text { prosthetic joint } \\
\text { infection }\end{array}$ & MSSA & 8 & flucloxacillin & $\begin{array}{l}\text { unplanned } \\
\text { readmission }\end{array}$ & $\begin{array}{l}\text { surgery and prolongation of } \\
\text { antibiotic treatment with } \\
\text { flucloxacillin }\end{array}$ \\
\hline Male & 58 & $\begin{array}{l}\text { infection of a } \\
\text { vascular } \\
\text { prosthesis }\end{array}$ & $\begin{array}{l}\text { Staphylococcus } \\
\text { lugdunensis and } \\
\text { epidermidis, } \\
\text { Corynebacterium } \\
\text { spp. }\end{array}$ & 7 & flucloxacillin & $\begin{array}{l}\text { unplanned } \\
\text { readmission }\end{array}$ & $\begin{array}{l}\text { surgery and change of antibiotic } \\
\text { adapted to new culture results }\end{array}$ \\
\hline Female & 54 & $\begin{array}{l}\text { iliac bone infection } \\
\text { post-biopsy }\end{array}$ & MRSA & 16 & vancomycin & $\begin{array}{l}\text { unplanned } \\
\text { readmission }\end{array}$ & $\begin{array}{l}\text { surgery and change of antibiotic } \\
\text { adapted to new culture results }\end{array}$ \\
\hline Male & 54 & pelvic abscess & $\begin{array}{l}\text { polymicrobial } \\
\text { infection }\end{array}$ & 11 & cefepime & $\begin{array}{l}\text { unplanned } \\
\text { readmission }\end{array}$ & $\begin{array}{l}\text { palliative care and prolongation of } \\
\text { antibiotic treatment; death due } \\
\text { to oncological disease }\end{array}$ \\
\hline Male & 50 & $\begin{array}{l}\text { osteitis of the } \\
\text { olecranon }\end{array}$ & MSSA & 15 & flucloxacillin & relapse & $\begin{array}{l}\text { surgery and new course of } \\
\text { flucloxacillin }\end{array}$ \\
\hline Male & 51 & $\begin{array}{l}\text { Port-A-Cath } \\
\text { infection }\end{array}$ & $\begin{array}{l}\text { Staphylococcus } \\
\text { capitis }\end{array}$ & 3 & vancomycin & relapse & $\begin{array}{l}\text { removal of Port-A-Cath and new } \\
\text { course of antibiotics }\end{array}$ \\
\hline Male & 58 & prostatitis & $\begin{array}{l}\text { Pseudomonas } \\
\text { aeruginosa }\end{array}$ & 24 & $\begin{array}{l}\text { piperacillin/ } \\
\text { tazobactam }\end{array}$ & relapse & $\begin{array}{l}\text { new course of piperacillin/ } \\
\text { tazobactam }\end{array}$ \\
\hline
\end{tabular}

favours the use of first-line antimicrobial agents. We thus expect a knock-on effect on cure rates and benefits from the perspective of antimicrobial stewardship. The main concern is that antibiotic degradation in such devices could exceed the recommended limit of $10 \%$ and that this could lead to treatment failures and/or an excess of adverse events due to possible toxic degradation products of the antibiotics.

In this study, we verified the circulating antibiotic plasma concentrations of patients treated by antibiotics administered continuously over $24 \mathrm{~h}$ via elastomeric pumps. As shown in Figure 1 the calculated free antibiotic plasma concentrations were above the ECOFF of the bacteria to be treated in $92 \%$ of the measurements ( $86 \%-99 \%$ depending on the antibiotics).

We chose to use the ECOFF values for this analysis, because the true MIC for the microorganisms was only known in a small number of patients. As the MICs for bacteria follow a Gauss-shaped curve, free antibiotic concentrations were above the actual MIC for the microorganisms in the vast majority of cases, even when plasma drug concentrations were measured slightly below the population target.

None of the patients with treatment failures had a low ratio of free antibiotic plasma concentrations/ECOFF. In addition, the intermittent administration of the same antibiotics at similar daily dosage would have resulted in a much less favourable pharmacokinetic profile, with antibiotic residual levels dropping frequently below the ECOFF values of the microorganisms.

As shown in Figure 1 there was significant intra-patient variability among the measured antibiotic plasma concentrations. Although random sampling time assumes a steady infusion rate, elastomeric pumps show a variable infusion rate, sometimes leading to premature completion of the infusion. ${ }^{11}$ Thus, blood concentrations measured early or late during the infusion period may be higher or lower than theoretically expected. Degradation of antibiotics in the elastomeric pumps could also have contributed to variations in antibiotic plasma concentrations depending on the time the blood was drawn. The time of the blood sampling was not recorded; therefore, it was not possible to verify whether lower plasma concentrations were systematically at the end of the infusion periods. Yet this antibiotic degradation in the pumps was shown to be at most limited for the antibiotics used. ${ }^{4}$

The proportion of favourable outcomes in this cohort was very high. Several groups have reported the cure rates of cohorts of OPAT patients. In a comprehensive review that examined the outcomes of global OPAT programmes, the cure rates reported in the included studies varied from $72.5 \%$ to $95 \% .{ }^{12}$ There are two main issues when comparing different studies. First, there are no common outcome definitions and the time of evaluation is often variable. Second, the case mix is very different between the cohorts; owing to significant heterogeneity of patients, some have a large proportion of patients with easy-to-treat infections such as skin and soft tissue infections, while others have a larger proportion of more difficult-to-treat infections such as bone and joint infections. In our cohort, the cure rate at 3 months after the end of treatment was $95 \%$, despite a proportion of joint and bone infections $>50 \%$. Patients were only considered cured if there were no more signs of infection at the end of antibiotic treatment and if there was no relapse or readmission to the hospital for the same infectious problem within 3 months. This definition of cure is more stringent than in any other studies to date, in which the outcome is usually evaluated at the end of the treatment.

Possible explanations for these good outcomes are the low age of the study population (median of 59 years), which is probably 
indicative of a population without multiple comorbidities, and the absence of MDR bacteria. In addition, it could also suggest high efficacy of continuous antibiotic infusion.

The effectiveness of continuous administration of antibiotics has only been investigated in the acute care setting and its superiority has not been demonstrated conclusively over the discontinuous administration of antibiotics. In a Cochrane Review including 29 studies, the median treatment duration was 9.8 days (414 days). ${ }^{13}$ In our study the median duration of treatment was 13 days and may have been more appropriate to show the benefit of continuous antimicrobial administration. Our results may even support the hypothesis that continuous antimicrobial administration could be particularly effective for deep, difficult-to-treat infections. For example, in this cohort, the successful outcome of the patients treated for the notoriously difficult-to-treat osteo-articular infections was $96 \%$ (70 of 73 patients). Other OPAT units treating populations of patients with a large percentage with bone and joint infections (as much as $43 \%-60 \%$ of them) have reported slightly less favourable outcomes with cure rates of $86 \%-93 \%{ }^{14-}$ 17 These data should prompt the initiation of a randomized trial comparing OPAT with continuous infusions versus OPAT with intermittent administration of antibiotics, to confirm formally the favourable outcomes of continuous OPAT with elastomeric devices.

Nowadays there is a trend towards shorter durations of intravenous antibiotic treatments as currently investigated for bone and joint infections in the OVIVA trial. ${ }^{18}$ The median duration of OPAT of 13 days in this study could be considered relatively long, considering that all patients had already received intravenous antibiotics during their hospital stay. The reasons for these relatively long intravenous treatment durations were not analysed in detail, but we postulate that many of our patients had particularly difficult-to-treat infections. We emphasize that we do not advocate prolonged treatments with intravenous antibiotics. For example, at our institution the recommended duration of intravenous treatment is 14 days for uncomplicated bone and joint infections, including prosthetic joint infections.

Sixteen (11\%), mostly minor, adverse events were observed. The adverse events were mostly expected side effects of the administered drugs. We did not observe adverse events suggesting hypersensitivity, for which the reported potentially toxic degradation products of the antibiotics could be blamed. In this observational study, adverse events were not associated with excessive or insufficient plasma antibiotic concentrations.

A limitation of this study is that the statistical power was insufficient to draw any firm conclusion regarding whether the ratio of free concentration over the ECOFF of the bacteria to be treated would be a predictor of either treatment failure or adverse reactions. Moreover, even if continuous infusion is generally expected to improve tissue distribution, antibiotic levels in tissues may differ from those of blood. Consequently, antibiotic plasma levels may not guarantee sufficient tissue exposure, which is known for high interpatient variability. A further limitation is the fact that free antibiotic concentrations were extrapolated from the fixed free fraction reference values available in the summary of product characteristics. The free fraction of drugs is, however, known to be difficult to establish and is characterized by significant inter-individual variability, being notably affected by patients' pathophysiological conditions, among other causes. Finally, the number of patients with an unfavourable outcome might have been underestimated. We only verified the occurrence of relapses and readmissions based on the records of our own hospital. Some patients may have consulted at other hospitals, although we do not think that this represents a significant number of patients.

In conclusion, these data suggest that OPAT using elastomeric pumps for the continuous administration of the four abovementioned antibiotics is efficacious and safe. Drug concentration measurements, considered a proxy for efficacy, confirm adequate circulating antibiotic exposures consistent with the observed high rate of therapeutic success.

\section{Funding}

This work was supported by an unrestricted grant from the Swiss General Internal Medicine Foundation.

\section{Transparency declarations}

None to declare.

\section{Supplementary data}

Tables S1 and S2 are available as Supplementary data at JAC Online.

\section{References}

1 Saillen L, Arensdorff L, Moulin E et al. Patient satisfaction in an outpatient parenteral antimicrobial therapy (OPAT) unit practising predominantly selfadministration of antibiotics with elastomeric pumps. Eur J Clin Microbiol Infect Dis 2017; 36: 1387-92.

2 Bing CM, Nowobilski-Vasilios A. Extended Stability for Parenteral Drugs. Bethesda, MD, USA: American Society of Health-System Pharmacists, 2013.

3 Jenkins A, Hills T, Santillo M et al. Extended stability of antimicrobial agents in administration devices. J Antimicrob Chemother 2017; 72: 1217-20.

4 Voumard R, Van Neyghem N, Cochet C et al. Antibiotic stability related to temperature variations in elastomeric pumps used for outpatient parenteral antimicrobial therapy (OPAT). J Antimicrob Chemother 2017; 72: 1462-5.

5 Arensdorff L, Boillat-Blanco N, Decosterd L et al. Adequate plasma drug concentrations suggest that amoxicillin can be administered by continuous infusion using elastomeric pumps. J Antimicrob Chemother 2017; 72: 2613-15.

6 EUCAST. Antimicrobial Wild Type Distributions of Microorganisms. https:// mic.eucast.org/Eucast2/.

7 FDA. Guidances for Industry and Investigators on Safety Reporting Requirements for Investigational New Drug Applications and Bioavailability/ Bioequivalence Studies, and a Small Entity Compliance Guide; Availability. https://www.federalregister.gov/documents/2012/12/20/2012-30651/gui dances-for-industry-and-investigators-on-safety-reporting-requirementsfor-investigational-new.

8 National Institutes of Health, National Cancer Institute. Common Terminology Criteria for Adverse Events (CTCAE). https://ctep.cancer.gov/ protocolDevelopment/electronic_applications/docs/CTCAE_v5_Quick_ Reference_8.5x11.pdf.

9 Tice AD, Rehm SJ, Dalovisio JR et al. Practice guidelines for outpatient parenteral antimicrobial therapy. IDSA guidelines. Clin Infect Dis 2004; 38: 1651-72.

10 Chapman AL, Seaton RA, Cooper MA et al. Good practice recommendations for outpatient parenteral antimicrobial therapy (OPAT) in adults in the UK: a consensus statement. J Antimicrob Chemother 2012; 67: 1053-62. 
11 Ackermann M, Maier S, Ing $\mathrm{H}$ et al. Evaluation of the design and reliability of three elastomeric and one mechanical infusers. J Oncol Pharm Pract 2007; 13: 77-84.

12 MacKenzie M, Rae N, Nathwani D. Outcomes from global adult outpatient parenteral antimicrobial therapy programmes: a review of the last decade. Int J Antimicrob Agents 2014; 43: 7-16.

13 Shiu J, Wang E, Tejani AM et al. Continuous versus intermittent infusions of antibiotics for the treatment of severe acute infections. Cochrane Database Syst Rev 2013; issue 3: CD008481.

14 Cox AM, Malani PN, Wiseman SW et al. Home intravenous antimicrobial infusion therapy: a viable option in older adults. J Am Geriatr Soc 2007; 55: 645-50.
15 Baharoon S, Almodaimeg $\mathrm{H}$, Al Watban $\mathrm{H}$ et al. Home intravenous antibiotics in a tertiary care hospital in Saudi Arabia. Ann Saudi Med 2011; 31: 457-61.

16 Kieran J, O'Reilly A, Parker J et al. Self-administered outpatient parenteral antimicrobial therapy: a report of three years experience in the Irish healthcare setting. Eur J Clin Microbiol Infect Dis 2009; 28: 1369-74.

17 Upton A, Ellis-Pegler RB, Woodhouse A. Outpatient parenteral antimicrobial therapy (OPAT): a review of experience at Auckland Hospital. N Z Med J 2004; 117: U1020.

18 Li HK, Scarborough M, Zambellas R et al. Oral versus intravenous antibiotic treatment for bone and joint infections (OVIVA): study protocol for a randomised controlled trial. Trials 2015; 16: 583-95. 